\title{
Spontaneous Arrest of Sporadic Spinal Hemangioblastoma Growth after Postoperative Nodular Recurrence: Case Report
}

\author{
Adam Y. Li ${ }^{1}$, Alexander F. Post ${ }^{1}$, Jennifer B. Dai ${ }^{1}$, Tanvir F. Choudhri ${ }^{1}$ \\ 1. Neurosurgery, The Icahn School of Medicine at Mount Sinai, New York, USA \\ $\square$ Corresponding author: Adam Y. Li, adam.li@icahn.mssm.edu \\ Disclosures can be found in Additional Information at the end of the article
}

\section{Abstract}

Hemangioblastomas are rare, slow-growing, highly vascularized tumors of the central nervous system which often occur in the spinal cord. When presenting as sporadic, isolated tumors without Von-Hippel Lindau disease, they are curable through surgery with a low rate of recurrence. Tumor recurrence in these cases is usually associated with prior subtotal resection. However, to the best of our knowledge, cases of recurrent, sporadic spinal hemangioblastoma have not been reported to spontaneously arrest without intervention or symptoms. We report a patient who underwent an initial complete resection of a cervical spinal hemangioblastoma, a subtotal resection of tumor recurrence four and a half years later, and nine years of neurologic and radiographic stability with no additional interventions.

Categories: Neurosurgery, Oncology

Keywords: hemangioblastoma, spine, sporadic, recurrence, spontaneous arrest

\section{Introduction}

Hemangioblastomas are rare, slow-growing, highly vascularized tumors of the central nervous system most commonly found in the posterior cranial fossa and spinal cord [1]. The only known genetic etiology is the loss of functional Von Hippel-Lindau (VHL) tumor suppressor, in VHL disease, which is seen in $25 \%$ of hemangioblastomas. Sporadic, isolated spinal hemangioblastomas, without VHL mutation, typically present later in life as solitary lesions, cause more severe neurological deficits, and are often curable through surgery [2].

Received 08/21/2018

Review began 09/02/2018 Review ended 09/25/2018 Published 09/28/2018

(C) Copyright 2018

Li et al. This is an open access article distributed under the terms of the

Creative Commons Attribution License CC-BY 3.0., which permits unrestricted use, distribution, and reproduction in any medium, provided the original author and source are credited.
Recurrence of sporadic spinal hemangioblastomas is primarily associated with subtotal resection. To the best of our knowledge, there have been 13 described cases to date of sporadic spinal hemangioblastomas recurring postoperatively and requiring repeat surgery for symptomatic deficits [3-8]. However, no reported case of recurrent and sporadic spinal hemangioblastoma has spontaneously arrested after subtotal resection requiring no further surgical intervention. Here, the authors present the case of a 72-year-old woman who underwent two resections of recurrent cervical hemangioblastoma. Recurrence after the second operation spontaneously arrested and has remained stable for nine years.

\section{Case Presentation}

A 72-year-old woman presented with nine months of neck pain and left upper extremity pain and numbness, three months of right upper extremity pain, and more recent weakness in the

How to cite this article

Li A Y, Post A F, Dai J B, et al. (September 28, 2018) Spontaneous Arrest of Sporadic Spinal Hemangioblastoma Growth after Postoperative Nodular Recurrence: Case Report. Cureus 10(9): e3380. DOI 10.7759/cureus. 3380 


\section{Cureus}

left arm. She also had a long history of urinary stress incontinence with no changes in bowel or bladder function during the nine months of symptoms preceding her presentation. Physical examination was notable for 4/5 triceps strength, effort-limited 4+/5 left grip strength and symmetric reflexes. Magnetic resonance imaging (MRI) revealed a cervical intramedullary syrinx from C5-C7 with a nodule suggestive of a hemangioblastoma (Figure 1).
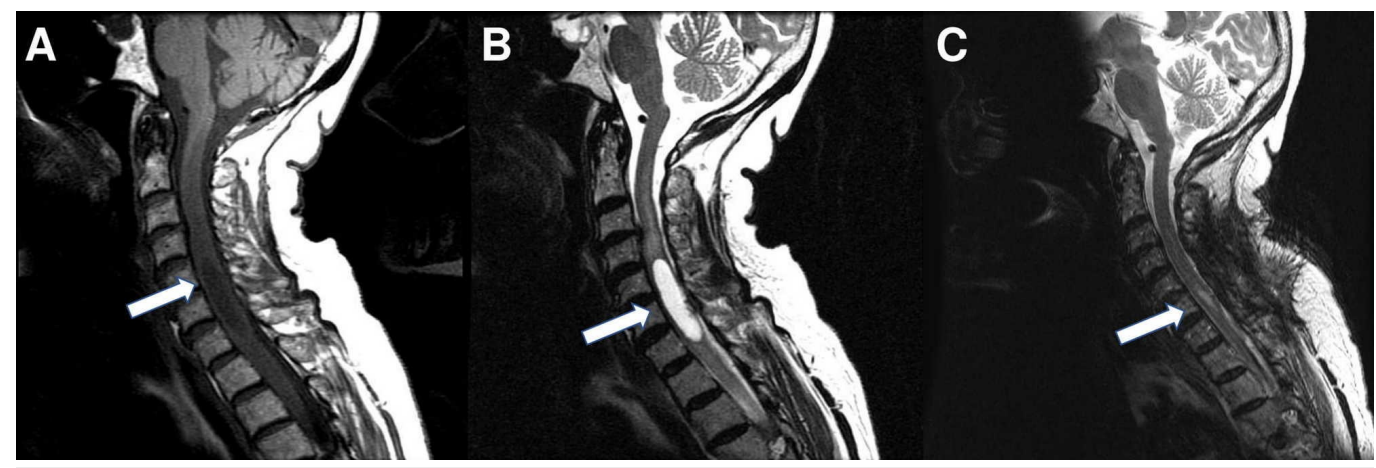

\section{FIGURE 1: Preoperative and postoperative imaging of cervical intramedullary hemangioblastoma with associated cyst}

Magnetic resonance (MR) images showing a cervical syrinx and nodule on (A) preoperative sagittal T1-spin echo (SE) and (B) T2-fast recovery fast spin echo (FRFSE). (C) Postoperative sagittal T2 MR image after removal of the hemangioblastoma with decompression of the syrinx.

She underwent uncomplicated elective $\mathrm{C} 5-\mathrm{C} 7$ laminectomies and resection of the nodule. Pathological testing confirmed the lesion to be a hemangioblastoma.

Imaging performed seven months postoperatively revealed abnormal hyperintensity of the left posteromedial spinal cord at C6 with a small, nodular, enhancing lesion of the most posterior portion of this abnormality. Subsequent imaging showed a progressive increase in the size of this lesion, and imaging four years postoperatively revealed increased nodular enhancement and recurrence of the syrinx (Figure 2). Four years postoperatively, she was also diagnosed with complex regional pain syndrome.

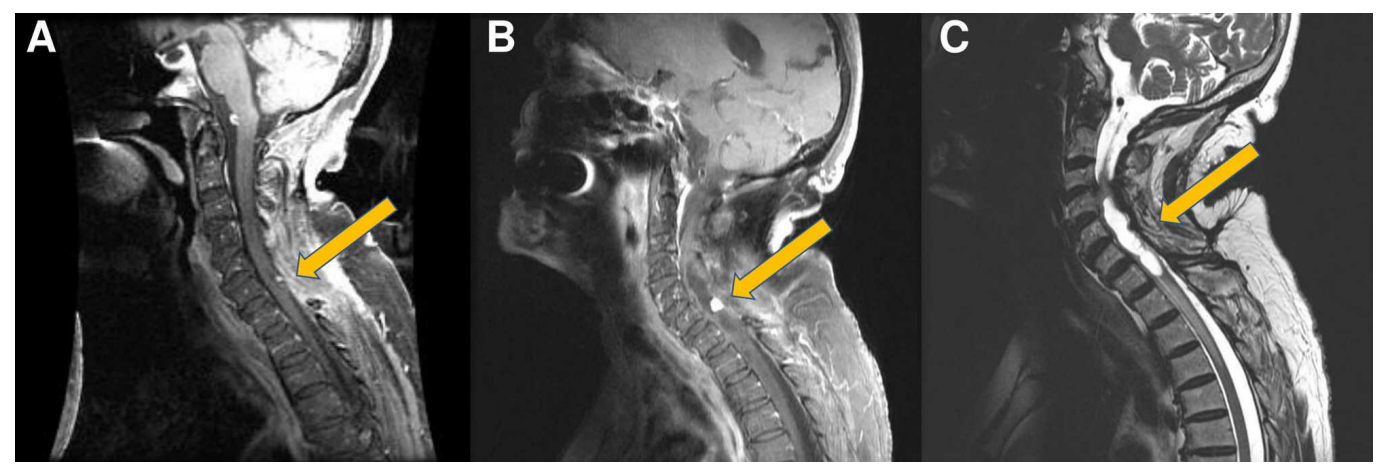

FIGURE 2: Recurrence of the hemangioblastoma and syrinx

(A) Seven-month postoperative, fat-suppressed magnetic resonance imaging (MRI) with contrast shows a small enhancing lesion at C6; (B) four-year postoperative T1-fluid-attenuated inversion recovery (FLAIR) MRI with contrast; (C) MRI fast recovery fast spin echo (FRFSE) images show lesion growth with the development of a syrinx. 


\section{Cureus}

Four and a half years after her index surgery, increasing enhancement size prompted additional surgery, including extension laminotomies/laminectomy from C3-T1 and resection of the pathology-proven hemangioblastoma. Imaging two months after the second surgery showed a punctate focus of enhancement at the dorsal aspect of C6, suspicious for residual or recurrent tumor. Subsequent annual imaging, with the most recent scan nine years after the second operation, has shown no significant changes in the focus of enhancement at C6 (Figure 3). No further interventional treatment has been performed.

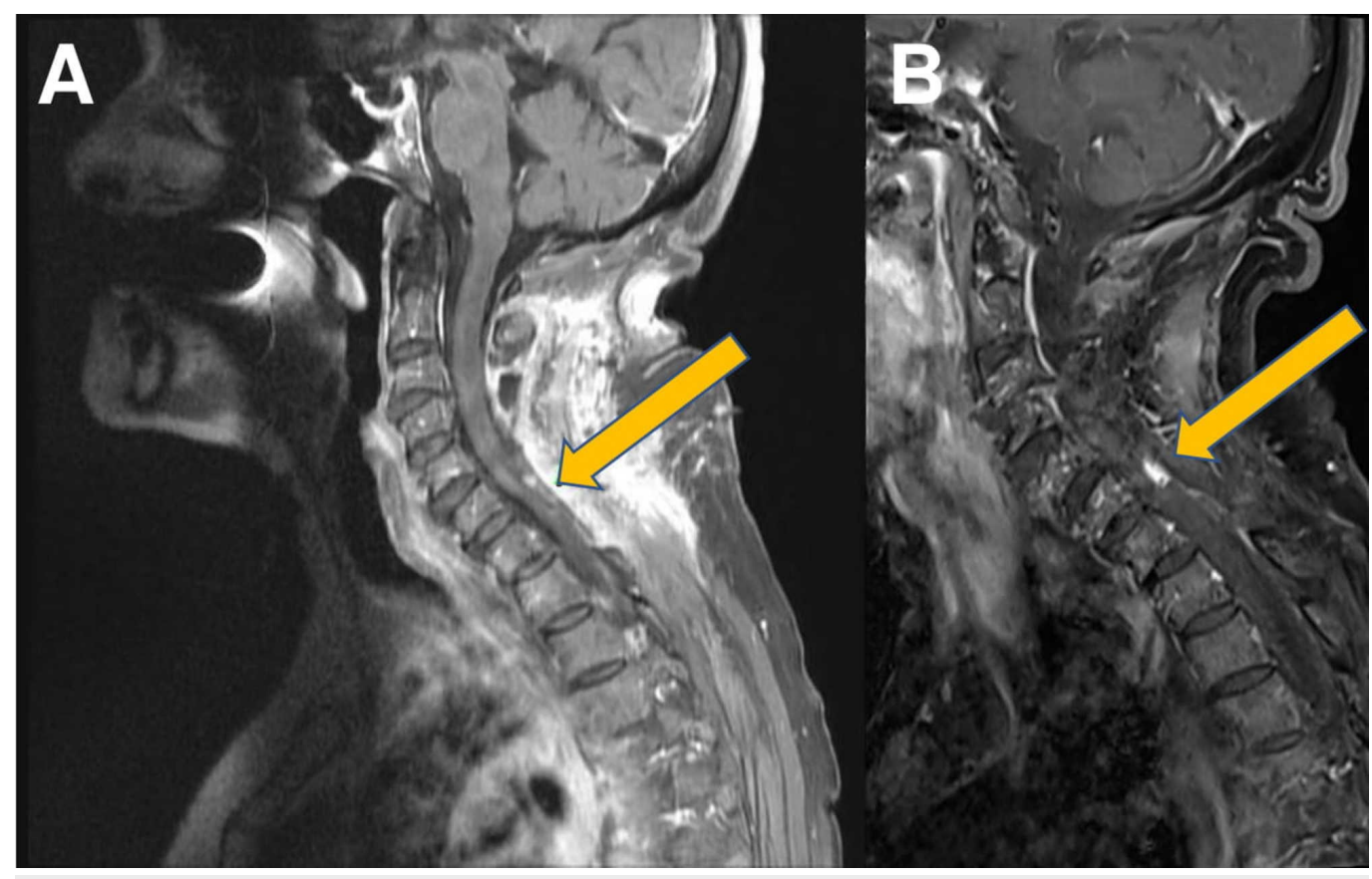

FIGURE 3: Postoperative nodule and lack of major postoperative growth

(A) Fluid-attenuated inversion recovery magnetic resonance imaging (FLAIR MRI) with contrast (four and a half years after initial operation, two months after second operation); (B) FLAIR MRI with contrast (13.5 years after initial operation, nine years after second operation)

\section{Discussion}

Hemangioblastomas are rare, highly vascularized tumors of the central nervous system accounting for $2-8 \%$ of intramedullary tumors [1]. Of patients with hemangioblastomas, the spine is the second most common site for disease, as $80 \%$ of hemangioblastoma patients develop lesions in the posterior cranial fossa and $20 \%$ of patients develop lesions in the spine [9]. Overall, understanding of the pathogenesis and role of mutated genes in the development of spinal hemangioblastomas remains limited.

One known causative factor of spinal hemangioblastomas is Von-Hippel Lindau disease caused by mutation or loss of the VHL gene. VHL encodes an E3 ubiquitin ligase that targets hypoxiainducible factor $1 \alpha($ HIF $1 \alpha)$, which is involved in the regulation of cell metabolism and vascular proliferation [1]. Mutation of VHL is present in approximately $25 \%$ of hemangioblastoma patients [1], and 88\% of hemangioblastoma patients with VHL present with spinal disease [6]. The role of VHL, as well as vascular proliferation in disease progression, makes anti-HIF1 $\alpha$ drugs and vascular endothelial growth factor (VEGF) inhibitors, such as the angiogenesis inhibitor bevacizumab, potential treatments for spinal hemangioblastomas in the setting of VHL disease. Anti-VEGF therapies, anti-HIF1 $\alpha$ therapies, IFN-alfa-2a, and thalidomide have 


\section{Cureus}

shown varying levels of therapeutic value in the treatment of VHL-associated hemangioblastomas $[1,10]$.

Sporadic hemangioblastomas have no known causative factors. Only $20 \%$ of these lesions occur in the spinal cord, and they have a different presentation when compared to VHL-associated lesions [6]. Sporadic disease typically presents later in life (4th vs 3rd decade), leads to more severe neurological deficits, and usually presents as a single larger lesion (in contrast to smaller multiple VHL-associated lesions). Typically, they are distributed in the cervical and thoracic spine (compared to all spine levels with VHL disease), and significantly improve after surgery (while VHL lesions do not and often recur) (Table 1) [6].

\section{Types of Hemangioblastomas}

Prevalence of spinal lesions

Known genetic mutation

Age at presentation

Neurological status at diagnosis

Tumor number

Tumor size

Tumor distribution

Surgical outcome

Factors affecting surgical outcome

New development of lesions

Potential new therapies

\section{Sporadic Disease}

$20 \%$

None

$30-40$

Mild to moderate deficits

Single

Large

Cervical and thoracic spine

Significant improvement

Partial removal of lesions

$6.25 \%-20 \%$ recurrence rate

None

\section{VHL-associated Disease}

$88 \%$

VHL tumor suppressor

$40-50$

No symptoms to mild deficits

Multiple

Small

All spine levels

No significant improvement

Partial removal of lesions

One-third of patients every two years

HIF1 $\alpha$ and VEGF inhibitors

\section{TABLE 1: Common clinical characteristics of sporadic and VHL-associated hemangioblastomas}

VHL: Von-Hippel Lindau; HIF1 $\alpha$; hypoxia-inducible factor 1 $\alpha$; VEGF: vascular endothelial growth factor

$[1,2,6]$

For both sporadic and VHL-associated hemangioblastomas, recurrence is associated with subtotal resection of lesions; however, the recurrence is rare in sporadic disease while onethird of patients with VHL-associated disease will recur every two years [6]. Overall, the rate of recurrence in sporadic disease has been reported between $6.25 \%$ and $20 \%$ [2]. Other than a VHL mutation, the clinical and molecular causes of hemangioblastoma proliferation are unknown. In our case, without VHL disease being present, it is unclear why the second recurrence spontaneously arrested after subtotal resection and no additional intervention. Interestingly, our patient was diagnosed with complex regional pain syndrome (CRPS) a year before her second operation. Various changes in genetics, inflammation signals, and circulating catecholamines have been known to affect CRPS, and it is unknown whether these factors 
played a role in affecting the growth of her sporadic hemangioblastoma [11]. Since sporadic spinal hemangioblastomas have been reported to recur up to 15 years postoperatively [2], it is still possible that symptomatic changes may occur. Therefore, future continued monitoring is needed. If the lesion continues to remain stable, it will indicate that subtotal resection of sporadic hemangioblastoma during surgery for recurrence does not always lead to significant growth or symptomatic disease.

\section{Conclusions}

Recurrence of sporadic hemangioblastomas occurs less frequently than VHL-associated hemangioblastomas. When sporadic hemangioblastomas do recur, they are usually associated with a subtotal resection. To the best of our knowledge, the sporadic recurrent hemangioblastoma presented here is the first reported case to spontaneously arrest without intervention. The mechanism of spontaneous arrest is unknown. Our patient was diagnosed with complex regional pain syndrome one year before her second operation, and it is unknown whether the CRPS contributed to the arrest of the tumor.

\section{Additional Information Disclosures}

Human subjects: Consent was obtained by all participants in this study. Conflicts of interest: In compliance with the ICMJE uniform disclosure form, all authors declare the following:

Payment/services info: All authors have declared that no financial support was received from any organization for the submitted work. Financial relationships: All authors have declared that they have no financial relationships at present or within the previous three years with any organizations that might have an interest in the submitted work. Other relationships: All authors have declared that there are no other relationships or activities that could appear to have influenced the submitted work.

\section{References}

1. Karsy M, Guan J, Sivakumar W, et al.: The genetic basis of intradural spinal tumors and its impact on clinical treatment. Neurosurg Focus. 2015, 39:E3. 10.3171/2015.5.FOCUS15143

2. Sun HI, Özduman K, Usseli MI, et al.: Sporadic spinal hemangioblastomas can be effectively treated by microsurgery alone. World Neurosurg. 2014, 82:836-47.

10.1016/j.wneu.2014.05.024

3. Boström A, Hans FJ, Reinacher PC, et al.: Intramedullary hemangioblastomas: timing of surgery, microsurgical technique and follow-up in 23 patients. Eur Spine J. 2008, 17:882-86. 10.1007/s00586-008-0658-1

4. Park CH, Lee CH, Hyun SJ, et al.: Surgical outcome of spinal cord hemangioblastomas . J Korean Neurosurg Soc. 2012, 52:221-27. 10.3340/jkns.2012.52.3.221

5. Malis LI: Atraumatic bloodless removal of intramedullary hemangioblastomas of the spinal cord. J Neurosurg. 2002, 97:1-6. 10.3171/spi.2002.97.1.0001

6. Takai K, Taniguchi M, Takahashi H, et al.: Comparative analysis of spinal hemangioblastomas in sporadic disease and Von Hippel-Lindau syndrome. Neurol Med Chir (Tokyo). 2010, 50:56067. 10.2176/nmc.50.560

7. Shin DA, Kim SH, Kim KN, et al.: Surgical management of spinal cord haemangioblastoma . Acta Neurochir (Wien). 2008, 150:215-20. 10.1007/s00701-008-1396-6

8. Raghavan R, Krumerman J, Rushing EJ, et al.: Recurrent (nonfamilial) hemangioblastomas involving spinal nerve roots: case report. Neurosurgery. 2000, 47:1443-48. 10.1097/00006123200012000-00038

9. Ostrom QT, Gittleman H, Liao P, et al.: CBTRUS statistical report: primary brain and central nervous system tumors diagnosed in the United States in 2007-2011. Neuro Oncol. 2014, 16:iv1-63. 10.1093/neuonc/nou223

10. Capitanio JF, Mazza E, Motta M, et al.: Mechanisms, indications and results of salvage 


\section{Cureus}

systemic therapy for sporadic and von Hippel-Lindau related hemangioblastomas of the central nervous system. Crit Rev Oncol Hematol. 2013, 86:69-84.

10.1016/j.critrevonc.2012.10.001

11. Bruehl S: Complex regional pain syndrome. BMJ. 2015, 351:h2730. 10.1136/bmj.h2730 